\title{
Intelligent Multimedia at the Imagineering Quarter
}

\author{
Paul Mc Kevitt \\ Chair in Intelligent MultiMedia, University of Ulster, Northern Ireland \\ p.mckevitt@ulster.ac.uk
}

\begin{abstract}
Here we focus on research in Intelligent MultiMedia or MultiModal computing concerning the computer processing and understanding of perceptual signal and symbol input from at least speech, text and visual images, and then reacting to it, involving signal and symbol processing techniques from engineering, computer science, artificial intelligence and cognitive science. With IntelliMedia systems, people can interact in spoken dialogues with machines, querying about what is being presented and even their gestures and body language can be interpreted. Of particular interest is the mapping of inputs into, and outputs out of, semantic representations and this is what distinguishes Intelligent MultiMedia from traditional MultiMedia. We will demonstrate here software prototypes such as 'PlayPhysics', which uses computer games to teach physics to first year university students. PlayPhysics is a virtual learning environment for teaching physics which integrates research in Intelligent Tutoring Systems (ITSs), where students learn about concepts such as momentum by trying to get an astronaut back to his craft in time by determining optimal mass and velocity. PlayPhysics also gives detailed feedback online. Another prototype we have developed is 'MemoryLane', a mobile digital storytelling companion for older people. Reminders of the person's past, such as photos, video, favourite songs or poems (provided by the individual or their family) are input as text, image, moving image and sound, creating the material from which multimodal stories can be generated. Each story is different and MemoryLane can factor in any problems with the person's eyesight, hearing or dexterity and adapt the presentation accordingly (like making the text larger or reducing the amount of sound or images). Based also on preferences it allows the holder to select aspects they like and reject anything they wish to forget. All of this work falls within 'The Imagineering Quarter' within the City of Derry/Londonderry, Northern Ireland, comprising 5 neighbouring buildings of the North West Regional College (NWRC) ('Foyle', 'Strand', 'Lawrence') \& University of Ulster ('Foyle Arts', 'Computing') focussed on teaching, research $\&$ technology transfer with software demonstrators in Digital Creativity (digital storytelling, music, film, theatre, dance, art, design; games, virtual worlds) linking to The Nerve Centre, Verbal Arts Centre community centres, crossborder Letterkenny Institute of Technology (LYIT), local software industry \& access to Project Kelvin -- a secure high capacity dedicated broadband link (10 G. LanPhy) direct to Canada, USA, Europe \& rest of the island with a delay of only $2 \mathrm{~ms}$. Derry/Londoderry is First UK City of Culture, 2013 and a key contributor is The Imagineering Quarter.
\end{abstract}

